\title{
SÍNDROME DE HORNER TRAS AMIGDALECTOMÍA
}

\section{HORNER'S SYNDROME AFTER TONSILLECTOMY}

\author{
BLANCO CI ${ }^{1}$, BUZNEGO L ${ }^{1}$, MÉNDEZ M ${ }^{1}$
}

\section{RESUMEN}

Caso clínico: Una niña de 5 años desarrolló un síndrome de Horner derecho tras realizársele una amigdalectomía. Se describen las características clínicas e investigaciones realizadas.

Discusión: El síndrome de Horner está causado por una disminución en la inervación simpática del ojo, y se define por la aparición de ptosis y miosis. No existen muchas publicaciones en que se relacione el síndrome de Horner con una amigdalectomía a pesar de la frecuencia con que se realiza esta cirugía $(1,2)$.

Palabras clave: Pupila, anisocoria, síndrome de Horner, ptosis, parálisis oculosimpática.

\section{ABSTRACT}

Case report: A 5-year-old girl underwent a tonsillectomy and developed a right Horner's syndrome after the procedure: we present the clinical findings and the investigations performed.

Discussion: Horner's syndrome is caused by a decrease in sympathetic innervation to the eye, and the defining signs are ptosis and miosis. The reported incidence of Horner's syndrome following tonsillectomy is low, although it is one of the commonest operative procedures performed $(1,2)$ (Arch Soc Esp Oftalmol 2008; 83: 129-132).

Key words: Pupil, anisocoria, Horner's syndrome, ptosis, oculosympathetic paralysis.

\section{INTRODUCCIÓN}

El síndrome de Horner fue descrito por Johann Friedrich Horner en 1869; a veces se denomina síndrome de Bernard Horner o también parálisis oculosimpática. Se debe a la lesión de una de las cadenas simpáticas cervicales, afectando al mismo lado de la lesión. Se caracteriza por la tríada patognomónica de ptosis, miosis y enoftalmos, aunque también puede acompañarse de dishidrosis hemifacial y pérdida del reflejo cilioespinal. Se presenta un caso de síndrome de Horner tras amigdalectomía en una niña de 5 años.

\section{CASO CLÍNICO}

La paciente es una niña de 5 años, con historial de múltiples episodios de amigdalitis y otitis media, a la que se le había practicado en otro centro una amigdalectomía y adenoidectomía, bajo anestesia general según la técnica habitual, sin complicaciones y sin infiltración de anestesia local postoperatoria.

El postoperatorio inmediato cursa con normalidad, pero a los 3 días la paciente presenta fiebre y deterioro del estado general, observando los padres que la pupila del ojo izquierdo es de mayor tamaño

\footnotetext{
Recibido: 2/10/06. Aceptado: 28/11/07.

Servicio de Oftalmología. Complejo Hospitalario Universitario de Albacete SESCAM. Albacete. España.

${ }^{1}$ Licenciado en Medicina.

Correspondencia:

Cristina Blanco Marchite

C/. Alcalde Martínez de la Ossa, 1-5E

02001 Albacete

España

E-mail: cblancomar@yahoo.es
} 
que la del ojo derecho. La paciente ingresa en el servicio de pediatría de nuestro hospital que la remite al servicio de oftalmología donde se llegó al diagnóstico de mediastinitis y síndrome de Horner.

A la exploración oftalmológica se observa (fig. 1):

- Miosis ojo derecho con luz natural, con aumento de la anisocoria en la oscuridad: la pupila derecha se dilata menos y más despacio que la izquierda.

- Ptosis ojo derecho leve con discreta elevación del párpado inferior.

— El resto del examen oftalmológico es normal: no hay heterocromía de iris ni enoftalmos, los movimientos oculares son normales y el paciente está en ortotropia.

Se instila en ambos ojos colirio de cocaína al $10 \%$, observándose a los 15 minutos que la pupila derecha no reacciona, mientras que la izquierda, no afectada, se dilata, lo que confirma el diagnóstico de Síndrome de Horner (fig. 2).

A los 6 meses de evolución la ptosis ha cedido, persistiendo únicamente una discreta anisocoria (fig. 3).

\section{DISCUSIÓN}

La cadena oculosimpática está compuesta por tres neuronas (3):

- Los axones de la neurona de primer orden: desde el hipotálamo discurren por el troncoencéfalo para hacer sinapsis en el centro cilio-espinal de Budge, a nivel de C8-T2.

- Los axones de la neurona de segundo orden ascienden al vértice pulmonar, pasan alrededor de

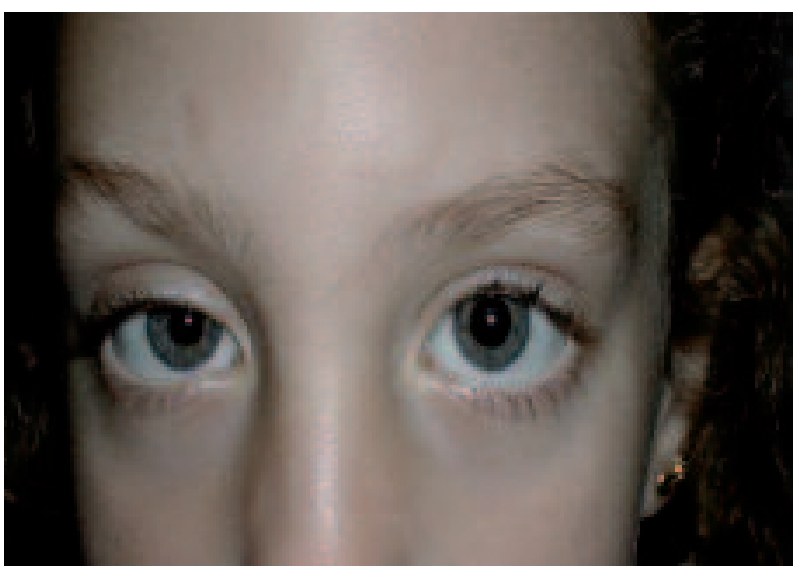

Fig. 1: Anisocoria en la exploración al ingreso.

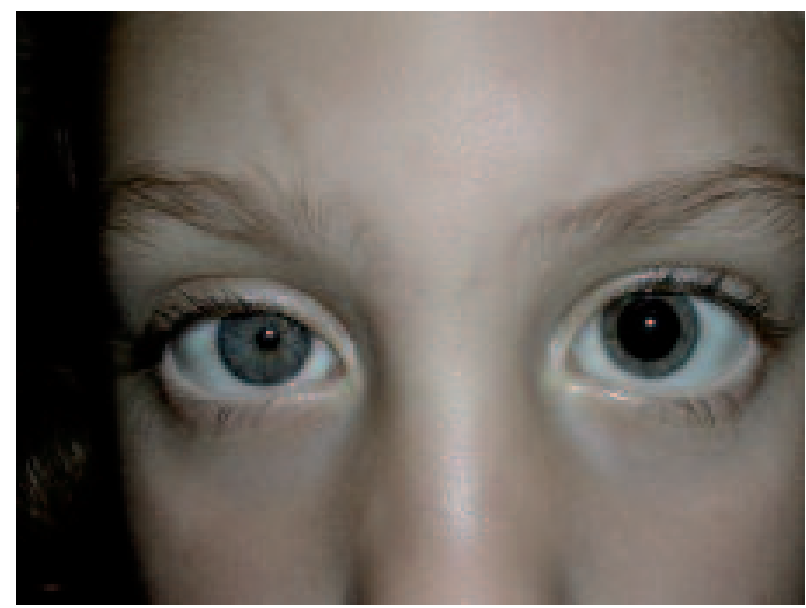

Fig. 2: Dilatación del ojo izquierdo tras la instilación de cocaína.

la arteria subclavia y hacen sinapsis en el ganglio cervical superior a nivel del ángulo de la mandíbula, 1,5 cm por detrás de la amígdala palatina.

- Desde la neurona de tercer orden (postganglionar) las fibras sudomotoras de la cara siguen a la carótida externa, y las fibras pupilares se hacen de nuevo intracraneales siguiendo a la carótida interna hasta el seno cavernoso, donde se incorporan a la rama oftálmica del trigémino hasta músculos dilatadores de la pupila, músculo de Müller del párpado superior y retractores del párpado inferior.

El síndrome de Horner es consecuencia de una parálisis oculosimpática, y se caracteriza por:

- Ptosis leve, con elevación del párpado inferior y enoftalmos aparente.

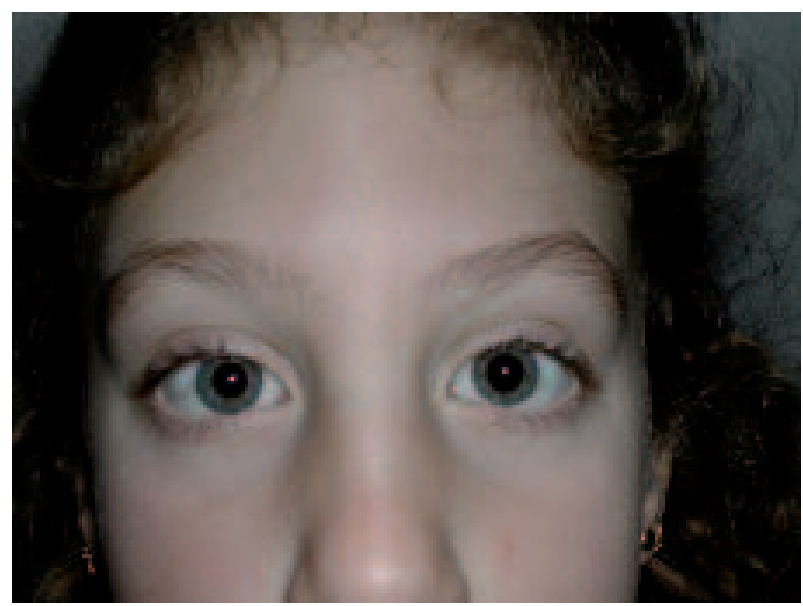

Fig. 3: Tras 6 meses de evolución se observa una mínima anisocoria. 
- Miosis (con reacciones pupilares normales) que produce una leve anisocoria, de $1 \mathrm{~mm}$ o menor, y con un característico retraso en la dilatación a la oscuridad: la anisocoria es mayor en la oscuridad y durante los primeros 5 segundos, reduciéndose a los 15-20 segundos.

- Anhidrosis según la localización de la lesión (poco evidente en las formas postganglionares).

- Heterocromía de iris en los casos congénitos, siendo más claro el iris afecto.

Pruebas farmacológicas:

Test de la cocaína al 2-10\%: dilata las pupilas normales inhibiendo la recaptación de la noradrenalina en la unión neuromuscular, sin embargo la pupila con parálisis simpática no dilata. Su utilidad mayor es en casos dudosos, porque sólo confirma la lesión oculosimpática, aunque no permite localizarla.

Test de la hidroxianfetamina (Paredrina): libera la noradrenalina en la unión neuromuscular provocando dilatación si la tercera neurona está intacta, y lo que permite discriminar así entre daño pre o postganglionar.

El test de la Hidroxianfetamina no está disponible en España, y además tiene un margen de error del $10 \%$ (3), que es mayor en niños por degeneración transináptica (4). Lo recomendable es realizar una TAC torácico y una RMN de cabeza y cuello en todos los pacientes con Horner sin clínica orientativa de un diagnóstico, aunque es sabido que el de causa postganglionar es con frecuencia benigno (3).

En niños con un síndrome de Horner agudo, de origen no traumático, se debe descartar un neuroblastoma (cervical o en mediastino) u otras masa ocupantes de espacio, teniendo en cuenta la alta frecuencia de falsos negativos de los niveles de catecolaminas en orina. Muchas veces están implicadas estructuras vasculares por lo que puede ser útil realizar angio-RMN (4).
En nuestro caso las pruebas realizadas (TAC torácico y niveles de ácido vanililmandélico en orina) fueron negativas, y la mediastinitis evolucionó favorablemente con tratamiento médico.

El síndrome de Horner ha sido descrito tras cirugía de amigdalectomía (2,5). Shissias sostiene que el daño se produce durante la intervención por la variabilidad y proximidad anatómica de la fosa amigdalar al ganglio cervical superior (2). Sin embargo, en un artículo recientemente publicado, Hobson sostiene que la causa del síndrome de Horner podría ser la infiltración anestésica realizada al final de la cirugía por difusión del fármaco infiltrado (5). En nuestra paciente esta causa queda descartada ya que no se realizó ningún tipo de infiltración.

Concluimos por tanto, que en nuestro caso el síndrome de Horner se ha debido al daño del ganglio cervical superior durante la cirugía de la amígdala palatina que se encuentra por detrás a sólo $1,5 \mathrm{~cm}$ $(2,5)$. El daño, por otra parte, fue reversible, y evolucionó hacia la casi completa resolución del cuadro.

\section{BIBLIOGRAFÍA}

1. Miller NR, Newman NJ. Disorders of papillary Function, Accommodation and Lacrimation. In: Miller NR, Newman NJ. Walsh \& Hoyt's Clinical Neuro-Ophthalmology. 5TH Ed. Baltimore: Williams \& Wilkins. 1999; 432-486.

2. Shissias CG, Golnik KC. Horner's syndrome after tonsillectomy. Am J Ophthalmol 1994; 117: 812-813.

3. Savino PJ, Danesh-Meyer HV. Horner's Syndrome. In: Savino PJ. Neuro-Ophthalmology: Color Atlas \& Synopsis of Clinical Ophthalmology. Columbus: Mc Graw-Hill. 2003; 223-227.

4. Burde RM, Savino PJ, Trobe JD. Anisocoria. In: Burde RM, Savino PJ, Trobe JD. Clinical Decisions in NeuroOphthalmology. 3RD Ed. St. Louis: Mosby. 2002; 246271.

5. Hobson JC, Malla JV, Kay NJ. Horner's syndrome following tonsillectomy. J Laryngol Otol 2006; 120: 800-801. 